\title{
FUNDAMENTAL SOLUTIONS FOR THE GENERAL LAMINATE PROBLEM WITH THE STRESS FUNCTION FORMALISM
}

\author{
STAVROS SYNGELLAKIS \\ Wessex Institute, UK.
}

\begin{abstract}
The linear coupled stretching-bending problem for general laminates is here formulated with the midplane stress function and the lateral deflection as independent field variables. A mathematical similarity between the two problems is achieved by introducing a re-arranged mid-plane strain tensor as one of the dependent variables. As a step towards a genuine boundary element solution for this problem, its fundamental solutions are derived using a Fourier transform approach. First, the transforms of the solutions are obtained in terms of the transform space variables and their inverses are deduced using complex integral calculus. Through the use of these fundamental solutions, boundary integral equations of the linear coupled stretching-bending problem are formulated without the presence of any irreducible domain integrals. Issues regarding the numerical implementation of this formulation are raised and discussed.
\end{abstract}

Keywords: boundary integral equations, coupled stretching-bending, fundamental solutions, general laminates, stress function formalism

\section{INTRODUCTION}

Composites have become attractive alternatives to traditional engineering materials because of their high specific stiffness and strength. They can be engineered to meet specific structural requirements through the choice of reinforcement and matrix materials, the volume fractions of fibre and matrix and the fabrication method. Laminated plates, in particular, are designed to have desired properties through the choice of layer orientation, number of layers in a given direction, thickness of individual layers, type of layer and the layer stacking sequence.

Laminated plates are modelled and analysed as elastic anisotropic solids. The anisotropy of a laminate depends on the anisotropy of its individual layers and the layer stacking sequence. The most complex form of such anisotropy, exhibited by general, non-symmetrically laminated plates, causes coupled stretching-bending response to applied in-plane and lateral forces.

Exact analyses of general laminates have been performed in cases of special material symmetry that eliminates a number of elastic constants without removing the coupling effect. Static solutions under simple loadings [1] were extended to free flexural vibration and buckling problems [2,3] under a variety of boundary conditions [4]. A post-buckling analysis under simple in-plane loading was also performed [5]. Numerical post-buckling solutions, based on finite element or finite difference methods and accounting for the stretching-bending coupling can also be found in the technical literature [6].

Through the boundary element method (BEM), complex plate geometries as well as loading and boundary conditions can be accommodated with the dimensionality of the problem being reduced by one. Numerical analysis schemes for general laminates, based on boundary element methodology, have been proposed although not fully implemented. Previous such analyses relied on the fundamental solutions of the two uncoupled, extensional and flexural problems [7]. The coupling of the two problems resulted in irreducible domain integrals and the proposed solution relied on the adoption of an iterative scheme. 
For a genuine BEM formulation, the fundamental solutions for the general laminate problem are essential. Explicit forms of such solutions have been derived [8] through an elaborate approach leading to expressions of considerable complexity. The mathematical basis of a BEM scheme relying on these solutions has also been developed [9].

The in-plane extensional problem can be formulated in two ways, that is, either in terms of the stress function $[1,3,5,7]$ or the in-plane displacements [2, 4] as field variables. Although the latter approach is more versatile in accounting for in-plane boundary conditions, the former is more mathematically elegant and possibly more suited to the case of stability problems when the applied contour traction is usually known. Fundamental solutions for the general laminate problem with the displacement formalism have been obtained using Fourier transforms [10]. In this paper, the same approach is applied to the problem formulated in terms of the stress function and the lateral deflection as field variables. The mathematical similarity between the extensional and flexural problems facilitates the derivation of fundamental solutions in very similar compact forms. Starting from a reciprocity relation, integral equations are derived by repeated applications of the Green's theorem. Introducing the derived fundamental solutions into these integral equations leads to a boundary element formulation without any irreducible domain integrals.

\section{LINEAR LAMINATE THEORY}

According to the classical lamination theory, the plate is assumed to be perfectly laminated consisting of an arbitrary number of discrete layers, each individual layer being homogeneous through its thickness and in a state of plane stress. The laminate is also assumed to deform according to Kirchhoff's assumptions for the bending of thin plates. According to this theory, the membrane forces $N_{\alpha \beta}$ and bending moments $M_{a, \beta}$ are related to the curvatures $\kappa_{a \beta}$ and mid-plane strains $\varepsilon_{a \beta}$ by

$$
\begin{aligned}
& N_{\alpha \beta}=A_{\alpha \beta \gamma \delta} \varepsilon_{\gamma \delta}+B_{a \beta \gamma \delta} \kappa_{\gamma \delta} \\
& M_{\alpha \beta}=B_{\alpha \beta \gamma \delta} \varepsilon_{\gamma \delta}+D_{a \beta \gamma \delta} \kappa_{\gamma \delta}
\end{aligned}
$$

where $A_{a \beta \gamma \delta}$ and $D_{a \beta \gamma \delta}$ are, respectively, the extensional and flexural rigidities, $B_{a \beta \gamma \delta}$ are extensional-flexural coupling coefficients and repeated Greek indices mean summation over their range, which is from 1 to 2 . It is evident from their derivation that all three material tensors are symmetric with respect to their pair of indices $\alpha \beta$ and $\gamma \delta$. Strains and curvatures are, respectively, related to the mid-plane displacements $u_{a}$ and deflection $w$ by

$$
\begin{gathered}
\varepsilon_{a \beta}=\frac{1}{2}\left(u_{a, \beta}+u_{\beta, a}\right) \\
\kappa_{\alpha \beta}=-w_{, a \beta}
\end{gathered}
$$

where a comma followed by a lower index indicates differentiation with respect to the corresponding co-ordinate. Finally, forces and moments should satisfy the equations of equilibrium

$$
\begin{aligned}
& N_{a \beta, \beta}+f_{a}=0 \\
& M_{a \beta, \alpha \beta}+q=0
\end{aligned}
$$

where $f_{a}$ is the body force assumed to be derivable from a potential function $\Phi$ according to

$$
f_{a}=-\Phi,{ }_{a}
$$

and $q$ is the lateral pressure. 
The field equations are complemented by the boundary conditions. Along the smooth portions of a boundary contour $\Gamma$ with normal and tangent unit vectors $\mathbf{n}$ and $\mathbf{s}$, respectively, the field variables should satisfy the conditions

$$
\begin{gathered}
p_{a}=n_{\beta} N_{a \beta} \\
V_{n}=n_{a} M_{\alpha \beta, \beta}+\frac{\partial M_{n s}}{\partial s} \\
M_{n}=n_{a} n_{\beta} M_{a \beta}
\end{gathered}
$$

where $p_{a}, V_{n}$ and $M_{n}$ are, respectively, the in-plane traction, shear force and bending moment, while, at any corner point $j$, the discontinuity jump of the twisting moment $M_{n s}$, given by

$$
M_{n s}=s_{a} n_{\beta} M_{\alpha \beta},
$$

should be equal to a concentrated force $C_{j}$.

If forces and moments are pre-scribed along the whole or part of $\Gamma, p_{a}, V_{n}, M_{n}$ and $C_{j}$ have known values there; alternatively, mid-plane displacements, deflection and deflection gradient $\theta_{n}$ may be pre-scribed along the whole or part of $\Gamma$, in which case $p_{a}, V_{n}, M_{n}$ and $C_{j}$ become the boundary unknowns of the problem.

\section{STRESS FUNCTION FORMALISM}

The problem can be re-formulated in terms of a stress function $F$ such that

$$
N_{\alpha \beta}=L_{\alpha \beta} F+\Phi \delta_{\alpha \beta}
$$

where $\delta_{a \beta}$ is the Kronecker delta and the operator $L_{a \beta}$ defined by

$$
L_{a, \beta}=\frac{\partial^{2}}{\partial x_{\kappa} \partial x_{\kappa}} \delta_{\alpha \beta}-\frac{\partial^{2}}{\partial x_{\alpha} \partial x_{\beta}}
$$

In-plane equilibrium, that is, eqn (5) with the body force given by eqn (7), is identically satisfied by the distributed internal forces given by the expressions of eqn (12). The stress function also needs to satisfy the compatibility condition imposed on the mid-plane strains, namely

$$
L_{\alpha \beta} \varepsilon_{\alpha \beta}=\bar{\varepsilon}_{\alpha \beta},_{\alpha \beta}=0
$$

where

$$
\bar{\varepsilon}_{\alpha \beta}=\varepsilon_{\kappa \kappa} \delta_{\alpha \beta}-\varepsilon_{\alpha \beta}
$$

is a re-arranged strain tensor introduced so that the compatibility condition, eqn (14), is mathematically similar to the second equilibrium eqn (6). Compatibility is imposed on the field variables by re-arranging the constitutive eqn (1). For this purpose, it is necessary to introduce the inverse of the extensional rigidity tensor $A_{\alpha \beta \gamma \delta}$ through

$$
A_{\alpha \beta \gamma \delta}^{-1} A_{\gamma \delta \lambda \mu}=\delta_{\alpha \lambda} \delta_{\beta \mu}
$$

Next, $N_{a \beta}$ and $\kappa_{a \beta}$ are substituted from eqns (12) and (4), respectively, into constitutive eqn (1), which is solved for $\varepsilon_{\alpha \beta}$ and the result substituted into eqn (15) to give

$$
\bar{\varepsilon}_{\alpha \beta}=\hat{A}_{\alpha \beta \gamma \delta} F,_{\gamma \delta}+\hat{B}_{\alpha \beta \gamma \delta} w,_{\gamma \delta}+\bar{A}_{\alpha \beta \kappa \kappa} \Phi
$$


where

$$
\begin{gathered}
\hat{A}_{\alpha \beta \gamma \delta}=A_{\kappa \kappa \lambda \lambda}^{-1} \delta_{\alpha \beta} \delta_{\gamma \delta}-A_{\alpha \beta \kappa \kappa}^{-1} \delta_{\gamma \delta}-A_{\kappa \kappa \gamma \delta}^{-1} \delta_{\alpha \beta}+A_{\alpha \beta \gamma \delta}^{-1} \\
\hat{B}_{\alpha \beta \gamma \delta}=\bar{A}_{\alpha \beta \lambda \mu} B_{\lambda \mu \gamma \delta}
\end{gathered}
$$

and

$$
\bar{A}_{\alpha \beta \gamma \delta}=A_{\kappa \kappa \gamma \delta}^{-1} \delta_{\alpha \beta}-A_{\alpha \beta \gamma \delta}^{-1}
$$

It should be noted that, among the tensors defined above, only that given by eqn (18) is symmetric with respect to the pair of indices $\alpha \beta$ and $\gamma \delta$.

The second constitutive equation also needs to be expressed in terms of the same field variables. Thus the expression for $\varepsilon_{a \beta}$ obtained by substituting $N_{a \beta}$ and $\kappa_{a \beta}$ from eqns (12) and (4), respectively, into eqn (1) and $\kappa_{\alpha \beta}$ from eqn (4) are substituted into eqn (2) to give

$$
M_{\alpha \beta}=\hat{B}_{\gamma \delta \alpha \beta} F,_{\gamma \delta}-\hat{D}_{\alpha \beta \gamma \delta} w,_{\gamma \delta}+\bar{B}_{\alpha \beta \kappa \kappa} \Phi
$$

where

$$
\hat{D}_{\alpha \beta \gamma \delta}=D_{\alpha \beta \gamma \delta}-B_{\alpha \beta \lambda \mu} A_{\lambda \mu \nu \xi}^{-1} B_{v \xi \gamma \delta}
$$

is the reduced flexural stiffness tensor and

$$
\bar{B}_{\alpha \beta \gamma \delta}=B_{\alpha \beta \lambda \mu} A_{\lambda \mu \gamma \delta}^{-1} .
$$

It is evident from eqn (22) that the reduced flexural stiffness tensor is symmetric with respect to the pair of indices $\alpha \beta$ and $\gamma \delta$ while the tensor defined by eqn (23) is not. However, it is evident from the new constitutive eqns (17) and (21) that the full elasticity tensor comprising the 4th-order tensors defined by eqns (18), (19), (22) and relating mid-plane strains and bending moments to the second derivatives of the field variables is symmetric.

The requirement that the mid-plane strains satisfy compatibility, eqn (14), and the bending moments satisfy equilibrium, eqn (6), leads to the 4th order differential equations

$$
\begin{gathered}
\hat{A}_{\alpha \beta \gamma \delta} F,_{\alpha \beta \gamma \delta}+\hat{B}_{\alpha \beta \gamma \delta} w,_{\alpha \beta \gamma \delta}=-\bar{A}_{\alpha \beta \kappa \kappa} \Phi,_{\alpha \beta} \\
\hat{B}_{\gamma \delta \alpha \beta} F,_{\alpha \beta \gamma \delta}-\hat{D}_{\alpha \beta \gamma \delta} w,_{\alpha \beta \gamma \delta}=-q-\bar{B}_{\alpha \beta \kappa \kappa} \Phi,_{\alpha \beta}
\end{gathered}
$$

The formulation is completed with the determination of the boundary conditions that replace eqn (8), that is, conditions satisfied by $F$ along the boundary contour. Using eqns (12), it has been shown that, at any point $Q\left(\bar{x}_{1}, \bar{x}_{2}\right)$ along the boundary,

$$
\begin{aligned}
& F=\int_{O}^{Q}\left[\left(x_{1}-\bar{x}_{1}\right) p_{2}-\left(x_{2}-\bar{x}_{2}\right) p_{1}\right] \mathrm{d} \Gamma \\
& \frac{\partial F}{\partial n}=-s_{1}(Q) \int_{O}^{Q} p_{1} \mathrm{~d} \Gamma-s_{2}(Q) \int_{O}^{Q} p_{2} \mathrm{~d} \Gamma
\end{aligned}
$$

where $O$ is an arbitrarily located origin. According to eqn (26), $F$ can be physically interpreted as the resultant moment about $Q$ of the traction over $O Q$. Similarly, eqn (27) describes the normal derivative of $F$ as the component of the resultant traction over $O Q$ in the direction -s at $Q$. It is evident from eqns (26) and (27) that $F$ and its normal derivative can be identified along $\Gamma$ only if traction has been specified along the whole boundary. If kinematic mid-plane 
conditions are imposed on parts of or the whole boundary, additional relations need to be adopted for a consistent formulation of the problem.

\section{FUNDAMENTAL SOLUTIONS}

A fundamental solution is defined as the response of an infinite domain to unit concentrated action, that is, force or moment. The force is applied at the source point $\mathrm{P}\left(\xi_{a}\right)$ while the response is determined at the field point $\mathrm{Q}\left(x_{a}\right)$ as illustrated in Fig. 1. Solutions to eqns (24) and (25) are therefore sought for mid-plane body forces

$$
f_{a}=\delta_{\lambda a} \delta(\mathbf{x}-\xi)
$$

that is, unit forces acting in directions parallel to the co-ordinate axes, a transverse unit point force

$$
q=\delta(\mathbf{x}-\xi)
$$

and a unit moment about the direction normal to unit in-plane vector $\mathbf{m}$ given by,

$$
q=\frac{\partial \delta(\mathbf{x}-\xi)}{\partial m(\xi)}=m_{a} \delta,_{a}(\mathbf{x}-\xi)
$$

These four solutions are represented by pairs of functions $\left(F_{\lambda}^{*}, w_{\lambda}^{*}\right)$ satisfying eqns (24) and (25) with right-hand sides given by eqn (28) for $\lambda=1,2$, by eqn (29) for $\lambda=3$ and by eqn (30) for $\lambda=4$.

\section{FOURIER TRANSFORMS}

In a two-dimensional space, the Fourier transform of a function $f(\mathbf{x})$ is given by [11]

$$
\hat{f}(\zeta)=\frac{1}{2 \pi} \int_{\Omega_{0}} f(\mathbf{x}) \mathrm{e}^{\mathrm{i} \zeta \cdot \mathrm{x}} \mathrm{d} \Omega
$$

where $\zeta$ is the two-dimensional independent variable in the Fourier space. For the purposes of the present analysis, it is important to note that the Fourier transform of the derivative $f$, is $\left(-\mathrm{i} z_{a}\right) \hat{f}(\zeta)$ where $\mathrm{i}$ is the unit imaginary number. Thus, taking the Fourier transforms of both sides of eqns (24) and (25) with the right-hand sides given by eqns (28), (29) and (30) converts the partial differential into algebraic operators comprising polynomials in $\zeta_{a}$.

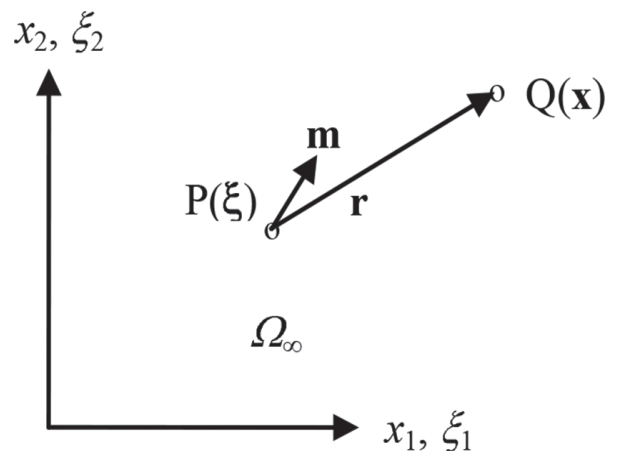

Figure 1: Infinite laminated plate under unit forces/moment at source point $\mathrm{P}(\xi)$. 
Thus, the Fourier transforms of the fundamental solutions for the field variables satisfy the equations

where

$$
\begin{aligned}
& \hat{A}_{\alpha \beta \gamma \delta} \zeta_{a} \zeta_{\beta} \zeta_{\gamma} \zeta_{\delta} \hat{F}_{\lambda}^{*}+\hat{B}_{a \beta \gamma \delta} \zeta_{a} \zeta_{\beta} \zeta_{\gamma} \zeta_{\delta} \hat{w}_{\lambda}^{*}=\frac{b_{1 \lambda}}{2 \pi} \mathrm{e}^{\mathrm{i} \zeta \cdot \xi} \\
& \hat{B}_{\gamma \delta \alpha \beta} \zeta_{a} \zeta_{\beta} \zeta_{\gamma} \zeta_{\delta} \hat{F}_{\lambda}^{*}-D_{a \beta \gamma \delta} \zeta_{a} \zeta_{\beta} \zeta_{\gamma} \zeta_{\delta} \hat{w}_{\lambda}^{*}=\frac{b_{2 \lambda}}{2 \pi} \mathrm{e}^{\mathrm{i} \zeta \cdot \xi}
\end{aligned}
$$

$$
\begin{gathered}
b_{1 \lambda}=-\mathrm{i} \hat{A}_{\lambda \beta \kappa \kappa} \zeta_{\beta}, b_{2 \lambda}=-\mathrm{i} \hat{B}_{\lambda \beta \kappa \kappa} \zeta_{\beta}, \lambda=1,2 \\
b_{13}=0, b_{23}=-1 \\
b_{14}=0, b_{24}=\mathrm{i} m_{\kappa} \zeta_{\kappa}
\end{gathered}
$$

The system of equations (31) and (32) can now be easily solved for the Fourier transforms of the fundamental solutions. The results take the form

$$
\begin{aligned}
& \hat{F}_{\lambda}^{*}=\frac{g_{\lambda}\left(\zeta_{1}, \zeta_{2}\right)}{\Delta\left(\zeta_{1}, \zeta_{2}\right)} \frac{\mathrm{e}^{\mathrm{i} \zeta \cdot \xi}}{2 \pi} \\
& \hat{w}_{\lambda}^{*}=\frac{h_{\lambda}\left(\zeta_{1}, \zeta_{2}\right)}{\Delta\left(\zeta_{1}, \zeta_{2}\right)} \frac{\mathrm{e}^{\mathrm{i} \zeta \cdot \xi}}{2 \pi}
\end{aligned}
$$

where

$$
\begin{gathered}
\Delta\left(\zeta_{1}, \zeta_{2}\right)=\left(\hat{A}_{a \beta \gamma \delta} \hat{D}_{\kappa \mu \nu \xi}+\hat{B}_{\kappa \mu \nu \xi} \hat{B}_{\gamma \delta \alpha \beta}\right) \zeta_{\alpha} \zeta_{\beta} \zeta_{\gamma} \zeta_{\delta} \zeta_{\kappa} \zeta_{\mu} \zeta_{\nu} \zeta_{\zeta}, \\
g_{\lambda}\left(\zeta_{1}, \zeta_{2}\right)=-\mathrm{i}\left(\hat{A}_{\lambda \beta \kappa \kappa} \hat{D}_{a \mu \gamma \delta}+\hat{B}_{\lambda \beta \kappa \kappa} \hat{B}_{a \mu \gamma \delta}\right) \zeta_{\alpha} \zeta_{\beta} \zeta_{\gamma} \zeta_{\delta} \zeta_{\mu} ; \lambda=1,2 \\
h_{\lambda}\left(\zeta_{1}, \zeta_{2}\right)=\mathrm{i}\left(\hat{B}_{\lambda \beta \kappa \kappa} \hat{A}_{a \mu \gamma \delta}-\hat{A}_{\lambda \beta \kappa \kappa} \hat{B}_{\gamma \delta a \mu}\right) \zeta_{\alpha} \zeta_{\beta} \zeta_{\gamma} \zeta_{\delta} \zeta_{\mu} ; \lambda=1,2 \\
g_{3}\left(\zeta_{1}, \zeta_{2}\right)=\hat{B}_{a \beta \gamma \delta} \zeta_{a} \zeta_{\beta} \zeta_{\gamma} \zeta_{\delta}, \\
h_{3}\left(\zeta_{1}, \zeta_{2}\right)=-\hat{A}_{a \beta \gamma \delta} \zeta_{a} \zeta_{\beta} \zeta_{\gamma} \zeta_{\delta}, \\
g_{4}\left(\zeta_{1}, \zeta_{2}\right)=-\mathrm{i} m_{\kappa} \hat{B}_{a \beta \gamma \delta} \zeta_{a} \zeta_{\beta} \zeta_{\gamma} \zeta_{\delta} \zeta_{\kappa}=-\mathrm{i} m_{\kappa} \zeta_{\kappa} g_{3}\left(\zeta_{1}, \zeta_{2}\right) \\
h_{4}\left(\zeta_{1}, \zeta_{2}\right)=\mathrm{i} m_{\kappa} \hat{A}_{a \beta \gamma \delta} \zeta_{a} \zeta_{\beta} \zeta_{\gamma} \zeta_{\delta} \zeta_{\kappa}=-\mathrm{i} m_{\kappa} \zeta_{\kappa} h_{3}\left(\zeta_{1}, \zeta_{2}\right)
\end{gathered}
$$

Thus $\Delta\left(\zeta_{1}, \zeta_{2}\right)$ is an 8 th order homogeneous polynomial in either $\zeta_{1}$ or $\zeta_{2}$ whose coefficients are expressed in terms of the material constants. Similarly, the functions $g_{\lambda}\left(\zeta_{1}, \zeta_{2}\right)$ and $h_{\lambda}\left(\zeta_{1}, \zeta_{2}\right)$ are homogeneous polynomials of 5 th order for $\lambda=1,2,4$ and of 4 th order for $\lambda=3$.

\section{INVERSE FOURIER TRANSFORMS}

The similarity of the expressions for the Fourier transforms of the stress function and the deflection suggests that the derivation of only one inverse transform needs to be described in detail. In this derivation, only the distinction between 4th and 5th order polynomials in the numerator needs to be made. 


\subsection{Stress function}

The inverse of the Fourier transform given by eqn (33) is written:

$$
F_{\lambda}^{*}(\mathbf{x}-\xi)=\frac{1}{2 \pi} \int_{\Omega_{\infty}} \hat{F}_{\lambda}^{*}(\zeta) \mathrm{e}^{-\mathrm{i} \zeta \cdot \mathrm{x}} \mathrm{d} \Omega_{\zeta}=\frac{1}{4 \pi^{2}} \int_{\Omega_{\infty}} \frac{g_{\lambda}\left(\zeta_{1}, \zeta_{2}\right)}{\Lambda\left(\zeta_{1}, \zeta_{2}\right)} \mathrm{e}^{-\mathrm{i} \zeta \cdot(\mathbf{x}-\xi)} \mathrm{d} \Omega_{\zeta}
$$

The integration on the right-hand sides of eqn (42) is performed by separation of variables:

$$
F_{\lambda}^{*}=\frac{1}{4 \pi^{2}} \int_{-\infty}^{\infty} \mathrm{e}^{-\mathrm{i} \zeta_{1} r_{1}} \mathrm{~d} \zeta_{1} \int_{-\infty}^{\infty} \frac{g_{\lambda}\left(\zeta_{1}, \zeta_{2}\right)}{\Delta\left(\zeta_{1}, \zeta_{2}\right)} \mathrm{e}^{-\mathrm{i} \zeta_{2} r_{2}} \mathrm{~d} \zeta_{2}
$$

where $r_{a}=x_{a}-\xi_{a}$. Integration with respect to $\zeta_{2}$ is performed using complex variable calculus [12]. For this purpose, a complex variable $z=\zeta_{2}+\mathrm{i} \zeta^{\prime}$ is introduced and a complex function $H(z)$ defined by

$$
H(z)=\frac{g_{\lambda}\left(\zeta_{1}, z\right)}{\triangle\left(\zeta_{1}, z\right)} \mathrm{e}^{-i r_{2} z}
$$

$H(z)$ is integrated around the contour shown in Fig. 2 comprising the segment of the real axis from $-R$ to $R$ and the semicircle $C_{R}$ in the upper half of the complex plane.

Thus, for any real value of $R$,

$$
\int_{-R}^{R} \frac{g_{\lambda}\left(\zeta_{1}, \zeta_{2}\right)}{\Delta\left(\zeta_{1}, \zeta_{2}\right)} \mathrm{e}^{-\mathrm{i} \zeta_{2} r_{2}} \mathrm{~d} \zeta_{2}+\int_{C_{R}} H(z) \mathrm{d} z=2 \pi \mathrm{i} \sum_{k} \operatorname{Res}\left(a_{k}\right)
$$

where $a_{k}$ represent the poles of $H(z)$ within the contour. It has been shown [12] that the contour integral on the left-hand side tends to zero as $R$ goes to infinity provided that $r_{2}<0$. As $R$ increases, the semicircle expands and eventually encompasses all the poles of $H(z)$. To determine the residues associated with these poles, it is noted that the 8 th order polynomial $\Delta\left(\zeta_{1}, z\right)$ has four pairs of distinct complex conjugate roots [13] which can be easily expressed in terms of $\zeta_{1}$ by introducing the parameter $\rho$ such that $z=\zeta_{2}=\rho \zeta_{1}$; this transforms eqn (35) into

$$
\Delta\left(\zeta_{1}, z\right)=\zeta_{1}^{8} \Delta(1, \rho)
$$

The roots of $\Delta(1, \rho)$ are represented by

$$
\rho_{k}=\rho_{k}^{\prime}+\mathrm{i} \rho_{k}^{\prime \prime}, \bar{\rho}_{k}=\rho_{k}^{\prime}-\mathrm{i} \rho_{k}^{\prime \prime}, \rho_{k}^{\prime \prime}>0 ; k=1,2,3,4
$$

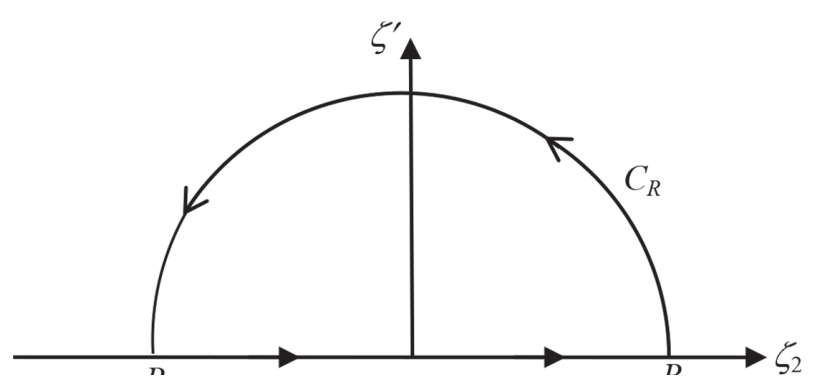

Figure 2: Contour for complex function integration. 
where a bar above a symbol represents the complex conjugate of the corresponding parameter. The denominator in eqn (44) can thus be written

$$
\Delta\left(\zeta_{1}, \zeta_{2}\right)=\Delta_{8} \prod_{i=1}^{4}\left(\zeta_{2}-\rho_{i} \zeta_{1}\right)\left(\zeta_{2}-\bar{\rho}_{i} \zeta_{1}\right)
$$

where $\Delta_{8}$ is the coefficient of $\rho^{8}$ which can be expressed explicitly in terms of material constants according to eqn (35). Before proceeding with the application of eqn (45), it is, at this stage, necessary to make the distinction between $\zeta_{1}$ being positive or negative.

(a) $\zeta_{1}>0$

In this case, $H(z)$ has four poles $\rho_{k} \zeta_{1}$ of order 1 , that is, simple poles, in the upper half-plane and the respective residues are

$$
\operatorname{Res}\left(\rho_{\kappa} \zeta_{1}\right)=\left[\left(z-\rho_{k} \zeta_{1}\right) H(z)\right]_{z=\rho_{k} \zeta_{1}}=\frac{g_{\lambda}\left(1, \rho_{k}\right) e^{-\mathrm{i} r_{2} \rho_{k} \zeta_{1}}}{2 \mathrm{i} \Delta_{8} \beta_{k} \zeta_{1}^{a}}
$$

where exponent $a$ in the denominator of the right-hand side is equal to either 2 or 3 , depending on whether $g_{\lambda}$ is a 5 th order or 4 th order polynomial, respectively, and

$$
\beta_{k}=\rho_{k}^{\prime \prime} \prod_{i=1, i \neq k}^{4}\left(\rho_{k}-\rho_{i}\right)\left(\rho_{k}-\bar{\rho}_{i}\right)
$$

Thus, from eqn (45)

$$
\int_{-\infty}^{\infty} \frac{g_{\lambda}\left(\zeta_{1}, \zeta_{2}\right)}{\Lambda\left(\zeta_{1}, \zeta_{2}\right)} \mathrm{e}^{-\mathrm{i} \zeta_{2} r_{2}} \mathrm{~d} \zeta_{2}=\frac{\pi}{\Delta_{8}} \sum_{k=1}^{4} \frac{g_{\lambda}\left(1, \rho_{k}\right) \mathrm{e}^{-\mathrm{i} r_{2} \rho_{k} \zeta_{1}}}{\beta_{k} \zeta_{1}^{a}}
$$

(b) $\zeta_{1}<0$

In this case, the complex conjugates of $\rho_{k}$, multiplied by $\zeta_{1}$, are the four simple poles of $H(z)$ in the upper half-plane; hence,

$$
\operatorname{Res}\left(\bar{\rho}_{k} \zeta_{1}\right)=\left[\left(z-\bar{\rho}_{k} \zeta_{1}\right) H(z)\right]_{z=\bar{\rho}_{k} \zeta_{1}}=\frac{g_{\lambda}\left(1, \bar{\rho}_{k}\right) e^{-\mathrm{i} \zeta_{1} r_{2} \bar{\rho}_{k}}}{-2 \mathrm{i} \Delta_{8} \bar{\beta}_{k} \zeta_{1}^{a}}
$$

and

$$
\int_{-\infty}^{\infty} \frac{g_{\lambda}\left(\zeta_{1}, \zeta_{2}\right)}{\Delta\left(\zeta_{1}, \zeta_{2}\right)} \mathrm{e}^{-\mathrm{i} \zeta_{2} r_{2}} \mathrm{~d} \zeta_{2}=-\frac{\pi}{\Delta_{8}} \sum_{k=1}^{4} \frac{g_{\lambda}\left(1, \bar{\rho}_{k}\right) \mathrm{e}^{-\mathrm{i} \zeta_{1} r_{2} \bar{\rho}_{k}}}{\bar{\beta}_{k} \zeta_{1}^{a}}
$$

It is thus possible to obtain the inverse transform given by eqn (43) over the whole range of $\zeta_{1}$ :

$$
F_{\lambda}^{*}=\frac{1}{4 \pi \Delta_{8}} \sum_{k=1}^{4} \frac{g_{\lambda}\left(1, \rho_{k}\right)}{\beta_{k}} \int_{0}^{\infty} \frac{\mathrm{e}^{-\mathrm{i} \zeta \nu_{1} \nu_{k}}}{\zeta_{1}^{a}} \mathrm{~d} \zeta_{1}-\frac{1}{4 \pi \Delta_{8}} \sum_{k=1}^{4} \frac{g_{\lambda}\left(1, \bar{\rho}_{k}\right)}{\bar{\beta}_{k}} \int_{-\infty}^{0} \frac{\mathrm{e}^{-i \zeta_{1} \bar{v}_{k}}}{\zeta_{1}^{a}} \mathrm{~d} \zeta_{1}
$$

where $v_{k}=r_{1}+\rho_{k} r_{2}$ while $a=2$ for $\lambda=1,2,4$ and $a=3$ for $\lambda=3$. By substituting $\zeta_{1}=s$ in the first integral of the right hand side of eqn (46) and $\zeta_{1}=-s$ in the second, it is possible to show that the second term is simply minus the complex conjugate of the first. This leads to

$$
F_{\lambda}^{*}=\frac{1}{2 \pi \Delta_{8}} \operatorname{Re}\left[\sum_{k=1}^{4} \frac{g_{\lambda}\left(1, \rho_{k}\right)}{\beta_{k}} \int_{0}^{\infty} \frac{\mathrm{e}^{-\mathrm{i} s v_{k}}}{s^{a}} \mathrm{~d} s\right]
$$


Since $r_{2}<0, \operatorname{Re}\left(-\mathrm{i} v_{k}\right)<0$; thus $\exp \left(-\mathrm{i} v_{k} s\right)$ is bounded as $s$ goes to infinity. Under this condition, integration by parts leads to

$$
\int_{0}^{\infty} \frac{\mathrm{e}^{-\mathrm{i} s v_{k}}}{s^{2}} \mathrm{~d} s=-\mathrm{i} v_{k} \int_{0}^{\infty} \frac{\mathrm{e}^{-\mathrm{i} s v_{k}}}{s} \mathrm{~d} s
$$

and

$$
\int_{0}^{\infty} \frac{\mathrm{e}^{-\mathrm{i} s v_{k}}}{s^{3}} \mathrm{~d} s=\frac{v_{k}^{2}}{2} \int_{0}^{\infty} \frac{\mathrm{e}^{-\mathrm{i} s v_{k}}}{s} \mathrm{~d} s
$$

Then the Cauchy principal value of the divergent integral on the right-hand side of eqns (47) and (48) can be obtained by making use of a property of the Laplace transforms [12], which gives

$$
\mathrm{P} \int_{0}^{\infty} \frac{\mathrm{e}^{-\mathrm{i} s v_{k}}}{s} \mathrm{~d} s=-\ln v_{k}
$$

Thus, finally, the fundamental solutions for the in-plane stress function is provided in the form of the real functions

$$
\begin{gathered}
F_{\lambda}^{*}=\frac{1}{2 \pi \Delta_{8}} \operatorname{Re}\left[\sum_{k=1}^{4} \frac{\mathrm{i} g_{\lambda}\left(1, \rho_{k}\right) v_{k} \ln v_{k}}{\beta_{k}}\right] ; \lambda=1,2,4 \\
F_{3}^{*}=-\frac{1}{4 \pi \Delta_{8}} \operatorname{Re}\left[\sum_{k=1}^{4} \frac{g_{3}\left(1, \rho_{k}\right) v_{k}^{2} \ln v_{k}}{\beta_{k}}\right]
\end{gathered}
$$

If $r_{2}>0$, for the contour integral over $C_{R}$ to tend to zero as $R$ goes to infinity, the semicircle should lie in the lower half-plane. The process described above is repeated leading to the same final result.

\subsection{Deflection}

The derivation of the fundamental solutions for the deflection follows exactly the same steps as that for the stress function. Thus, the final expressions are the same as those given by eqns (50) and (51) but with function $h_{\lambda}$ replacing $g_{\lambda}$, namely,

$$
\begin{gathered}
w_{\lambda}^{*}=\frac{1}{2 \pi \Delta_{8}} \operatorname{Re}\left[\sum_{k=1}^{4} \frac{\mathrm{i} h_{\lambda}\left(1, \rho_{k}\right) v_{k} \ln v_{k}}{\beta_{k}}\right] ; \lambda=1,2,4 \\
w_{3}^{*}=-\frac{1}{4 \pi \Delta_{8}} \operatorname{Re}\left[\sum_{k=1}^{4} \frac{h_{3}\left(1, \rho_{k}\right) v_{k}^{2} \ln v_{k}}{\beta_{k}}\right]
\end{gathered}
$$

\section{BOUNDARY INTEGRAL EQUATIONS}

BEM can now be applied to the analysis of a finite laminated plate, whose domain $\Omega$ is bounded by the contour $\Gamma$ as shown in Fig. 3. Apart for the in-plane body forces and the 
lateral pressure, the plate may also be subjected to various edge loads such as traction $\mathbf{p}$. A BEM solution scheme can be developed by formulating first the reciprocity relation

where

$$
\begin{aligned}
& \left.\int_{\Omega}\left(\bar{\varepsilon}_{\alpha \beta}-\bar{A}_{a \beta \kappa \kappa} \Phi\right) F^{\prime},_{\alpha \beta}+\left(M_{\alpha \beta}-\bar{B}_{a \beta \kappa \kappa} \Phi\right) w^{\prime},_{\alpha \beta}\right) \mathrm{d} \Omega \\
= & \int_{\Omega}\left[\left(\bar{\varepsilon}_{a \beta}^{\prime}-\bar{A}_{\alpha \beta \kappa \kappa} \Phi^{\prime}\right) F,_{\alpha \beta}+\left(M_{\alpha \beta}^{\prime}-\bar{B}_{\alpha \beta \kappa \kappa} \Phi^{\prime}\right) w,_{\alpha \beta}\right] \mathrm{d} \Omega
\end{aligned}
$$

$$
\left(\bar{\varepsilon}_{\alpha \beta}, F, M_{\alpha \beta}, w\right) \text { and }\left(\bar{\varepsilon}_{\alpha \beta}^{\prime}, F^{\prime}, M_{\alpha \beta}^{\prime}, w^{\prime}\right)
$$

are two equilibrium states due, respectively, to body force-lateral pressure combinations $(\mathbf{f}, q)$ and $\left(\mathbf{f}^{\prime} q^{\prime}\right)$. As such they satisfy compatibility, eqn (14), and equilibrium, eqn (6). This reciprocity relation is proven by taking into account the constitutive eqns (17) and (21) as well as the symmetries of material tensors.

Repeated integrations by parts and applications of Green's theorem transform eqn (54) into

$$
\begin{aligned}
& \int_{\Omega}\left(\bar{\varepsilon}_{\alpha \beta}{ }_{a \beta} F^{\prime}-\bar{\varepsilon}_{\alpha \beta}^{\prime},_{a \beta} F\right) \mathrm{d} \Omega+\int_{\Omega}\left(M_{\alpha \beta},_{a \beta} w^{\prime}-M_{\alpha \beta}^{\prime},_{\alpha \beta} w\right) \mathrm{d} \Omega \\
& +\int_{\Omega} \bar{A}_{a \beta \kappa \kappa}\left(\Phi,{ }_{\beta} F^{\prime},{ }_{a}-\Phi^{\prime},{ }_{\beta} F,{ }_{\alpha}\right) \mathrm{d} \Omega+\int_{\Omega} \bar{B}_{a \beta \kappa \kappa}\left(\Phi,{ }_{\beta} w^{\prime},{ }_{a}-\Phi^{\prime},{ }_{\beta} w,{ }_{a}\right) \mathrm{d} \Omega \\
& =I_{F}\left(F, F^{\prime}\right)+J_{F}\left(F, F^{\prime}\right)+I_{w}\left(w, w^{\prime}\right)+J_{w}\left(w, w^{\prime}\right)+I_{\Phi}\left(F, F^{\prime}, w, w^{\prime}\right)
\end{aligned}
$$

It is noted that the first domain integral on the left-hand side of eqn (55) always vanishes on account of strains satisfying compatibility eqn (14). The right-hand side of eqn (55) comprises contour integrals and corner jump terms defined by the equations

$$
\begin{aligned}
& I_{F}\left(F, F^{\prime}\right)=\int_{\Gamma}\left(\kappa_{n} F^{\prime}-\varepsilon_{s s} \frac{\partial F^{\prime}}{\partial n}+\varepsilon_{s s}^{\prime} \frac{\partial F}{\partial n}-\kappa_{n}^{\prime} F\right) \mathrm{d} \Gamma \\
& I_{w}\left(w, w^{\prime}\right)=\int_{\Gamma}\left(V_{n} w^{\prime}-M_{n} \frac{\partial w^{\prime}}{\partial n}+M_{n}^{\prime} \frac{\partial w}{\partial n}-V_{n}^{\prime} w\right) \mathrm{d} \Gamma \\
& I_{\Phi}\left(F, F^{\prime}, w, w^{\prime}\right)=\int_{\Gamma} n_{\beta}\left[\Phi\left(\bar{A}_{\alpha \beta \kappa \kappa} F^{\prime},{ }_{\alpha}+\bar{B}_{\alpha \beta \kappa \kappa} w^{\prime},_{a}\right)-\Phi^{\prime}\left(\bar{A}_{\alpha \beta \kappa \kappa} F,_{a}+\bar{B}_{\alpha \beta \kappa \kappa} w,_{\alpha}\right)\right] \mathrm{d} I \\
& \text { }
\end{aligned}
$$

Figure 3: Laminated plate of arbitrary shape under edge traction $\mathbf{p}$. 


$$
\begin{gathered}
J_{F}\left(F, F^{\prime}\right)=\sum_{j=1}^{K}\left[\bar{\varepsilon}_{n s} F^{\prime}-\bar{\varepsilon}_{n s}^{\prime} F\right]_{j} \\
J_{w}\left(w, w^{\prime}\right)=\sum_{j=1}^{K}\left[M_{n s} w^{\prime}-M_{n s}^{\prime} w\right]_{j}
\end{gathered}
$$

where $K$ is the number of corner points along $\Gamma$. In eqn (56),

$$
\varepsilon_{s s}=\bar{\varepsilon}_{n n}=\frac{\partial u_{s}}{\partial s}
$$

is the normal strain in the direction of $\mathbf{s}$, while

$$
\kappa_{n}=n_{\alpha} \bar{\varepsilon}_{\alpha \beta},_{\beta}+\frac{\partial}{\partial s}\left(s_{\alpha} n_{\beta} \bar{\varepsilon}_{\alpha \beta}\right)=\frac{\partial \bar{\varepsilon}_{n n}}{\partial n}+2 \frac{\partial \bar{\varepsilon}_{n s}}{\partial s}=-\frac{\partial^{2} u_{n}}{\partial s^{2}}
$$

represents the change of curvature of the boundary $\Gamma$ due to the normal in-plane displacement $u_{n}$. It is worth noting the $\varepsilon_{s s}$ and $\kappa_{n}$ are multiplied, respectively, by a force acting in the s-direction and a moment about an axis perpendicular to the plane of the plate; it is thus evident that these products represent work terms.

It is next assumed that the loading system $(\mathbf{f}, q)$ is the one actually applied while $\left(\mathbf{f}^{\prime} q^{\prime}\right)$ comprises the unit actions given by eqns (28), (29) and (30) causing the fundamental states derived in the previous section. Then, the non-vanishing domain integrals on the left-hand side of eqn (55) are reduced to the left-hand side expressions given below:

$$
\begin{aligned}
& \int_{\Omega}\left(M_{a \beta}{ }^{a \beta}{ } w_{\lambda}^{*}-M_{\lambda a \beta}^{*},{ }_{\alpha \beta} w\right) \mathrm{d} \Omega=\left\{\begin{array}{l}
-\int_{\Omega} q w_{\lambda}^{*} \mathrm{~d} \Omega \text { for } \lambda=1,2 \\
-\int_{\Omega} q w_{3}^{*} \mathrm{~d} \Omega+w(\xi) \\
-\int_{\Omega} q w_{4}^{*} \mathrm{~d} \Omega+\frac{\partial w(\xi)}{\partial m}
\end{array}\right. \\
& -\int_{\Omega} \bar{A}_{\alpha \beta \kappa \kappa}\left(f_{\beta} F_{\lambda}^{*},_{\alpha}-f_{\lambda \beta}^{*} F,_{a}\right) \mathrm{d} \Omega=\left\{\begin{array}{cc}
-\int_{\Omega} \bar{A}_{\alpha \beta \kappa \kappa} f_{\beta} F_{\lambda, \alpha}^{*} \mathrm{~d} \Omega+\bar{A}_{\alpha \lambda \kappa \kappa} F,_{\alpha}(\xi) & \text { for } \lambda=1,2 \\
-\int_{\Omega} \bar{A}_{a \beta \kappa \kappa} f_{\beta} F_{\lambda}^{*},{ }_{\alpha} \mathrm{d} \Omega & \text { for } \lambda=3,4
\end{array}\right. \\
& -\int_{\Omega} \bar{B}_{a \beta \kappa \kappa}\left(f_{\beta} w_{\lambda}^{*},{ }_{a}-f_{\lambda \beta}^{*} w,{ }_{a}\right) \mathrm{d} \Omega=\left\{\begin{array}{cc}
-\int_{\Omega} \bar{B}_{a \beta \beta \kappa \kappa} f_{\beta} w_{\lambda,{ }_{a}}{ }^{*} \mathrm{~d} \Omega+\bar{B}_{a \lambda \kappa \kappa} w,_{a}(\xi) & \text { for } \lambda=1,2 \\
-\int_{\Omega} \bar{B}_{a \beta \kappa \kappa} f_{\beta} w_{\lambda}^{*},{ }_{a} \mathrm{~d} \Omega & \text { for } \lambda=3,4
\end{array}\right.
\end{aligned}
$$

The domain integrals in the above expressions depend on known quantities, they can therefore be evaluated. The variable of each free term is transferred to the boundary where unit vector $\mathbf{m}$ becomes identical to unit normal $\mathbf{n}$; this generates a factor $k$, which equals $1 / 2$ if $\mathrm{P}(\xi)$ lies on a smooth segment of $\Gamma$. The boundary variable $F,{ }_{a}$ can be evaluated since

$$
F,_{\alpha}=n_{\alpha} \frac{\partial F}{\partial n}+s_{\alpha} \frac{\partial F}{\partial s}
$$


and both $F(s)$ and its normal derivative are given, respectively, by eqns (26) and (27). The boundary deflection and its normal derivative can be known or unknowns depending on the specified boundary conditions. Thus, the deflection gradient $w,{ }_{a}$ can either be evaluated or modelled along $\Gamma$ using

$$
w,_{\alpha}=n_{\alpha} \frac{\partial w}{\partial n}+s_{\alpha} \frac{\partial w}{\partial s}
$$

The contour integrals on the left-hand side of eqn (55) depend on boundary variables multiplied by kernels derivable from the fundamental solutions using differentiation and the constitutive equations. Four of the boundary variables are unknown and need to be modelled using boundary elements. Special modelling schemes are required for the corner variables if any of them are not known.

Thus, the final form of the boundary integral equations on which a BEM scheme could be based is

$$
\begin{aligned}
I_{F}\left(F, F_{\lambda}^{*}\right)+ & J_{F}\left(F, F_{\lambda}^{*}\right)+I_{w}\left(w, w_{\lambda}^{*}\right)+J_{w}\left(w, w_{\lambda}^{*}\right)+I_{\Phi}\left(F, F_{\lambda}^{*}, w, w_{\lambda}^{*}\right) \\
& +\int_{\Omega}\left(\bar{A}_{a \beta \kappa \kappa} f_{\beta} F_{\lambda}^{*},_{a}+\bar{B}_{a \beta \kappa \kappa} f_{\beta} w_{\lambda}^{*},_{a}+q w_{\lambda}^{*}\right) \mathrm{d} \Omega \\
= & \left\{\begin{array}{cc}
k\left[\bar{A}_{a \lambda \kappa \kappa} F,_{a}(\xi)+\bar{B}_{a \lambda \kappa \kappa} w,_{a}(\xi)\right] & \text { for } \lambda=1,2 \\
k w_{\lambda}(\xi) & \text { for } \lambda=3,4
\end{array}\right.
\end{aligned}
$$

where

$$
w_{3}(\xi)=w(\xi) \text { and } w_{4}(\xi)=\frac{\partial w}{\partial n}(\xi)
$$

\section{CONCLUDING REMARKS}

Fundamental solutions were derived in closed, compact and elegant form; to be practically useful, their parameters depending on the material constants need to be initially evaluated. For this purpose, tensor products such as those appearing in eqn (35)-(41) should be expanded to identify the coefficients of the respective polynomials and the roots of the 8th degree polynomial $\Delta(1, \rho)$ determined. With all their constant parameters known, their dependence on space co-ordinates is quite simple and their variation can be evaluated without high computational cost. From the nature of the roots of $\Delta(1, \rho)$ it is evident that complex number coding would be necessary in any computer implementation.

Expressions for the various components of the fundamental states appearing as weighting functions in domain and contour integrals should also be derived. The derivatives of the fundamental solutions also have simple forms although entering them into constitutive equations would generate longer expressions.

The potential of using the obtained fundamental solutions in BEM schemes for general laminates was demonstrated through the derivation of a set of four boundary integral equations involving four boundary unknowns. In contrast to the formulation based on just the three displacement components as field variables, the present one using the stress function and deflection generates integral equations in identical form and thus susceptible to a more efficient and flexible numerical manipulation. 
A computer-implemented BEM formulation solving the general laminate problem under any loading and boundary conditions is expected to present many challenges. Practically relevant simplifications are possible, such as the elimination of applied in-plane body forces from the formulation. Two issues that require rigorous mathematical treatment is the determination of the in-plane force potential for the fundamental states and the derivation of expressions for the free term coefficient $k$ when the source point is a corner point. The latter issue is obviated if discontinuous boundary elements are used adjacent to a contour corner.

As already pointed out, it is expedient to specify only traction boundary conditions along the boundary for this formulation to work without further analysis and modelling. This is usually the case with stability analyses but the geometric nonlinearity of such problems introduce additional analytical and numerical complexities. Similar challenges would be faced in attempts to extend the BEM scheme to dynamic problems.

\section{REFERENCES}

[1] Reissner, E. \& Stavsky, Y., Bending and stretching of certain types of heterogeneous aeolotropic elastic plates. Transactions of the ASME, Journal of Applied Mechanics, 28(3), pp. 402-408, 1961.

https://doi.org/10.1115/1.3641719

[2] Whitney, J.M. \& Leissa, A.W., Analysis of heterogeneous, anisotropic plates. Transactions of the ASME, Journal of Applied Mechanics, 36, pp. 261-266, 1969. https://doi.org/10.1115/1.3564618

[3] Whitney, J.M. \& Leissa, A.W., Analysis of a simply supported laminated anisotropic rectangular plate. AIAA Journal, 8(1), pp. 28-33, 1970. https://doi.org/10.2514/3.5601

[4] Whitney, J.M., The effect of boundary conditions on the response of laminated composites. Journal of Composite Materials, 4(2), pp. 192-203, 1970.

https://doi.org/10.1177/002199837000400205

[5] Harris, G.Z., The buckling and post-buckling behaviour of composite plates under biaxial loading. International Journal of Mechanical Sciences, 17, pp. 187-202, 1975. https://doi.org/10.1016/0020-7403(75)90052-1

[6] Noor, A.K., Mathers, M.D. \& Anderson, M.S., Exploiting symmetries for efficient postbuckling analysis of composite plates. AIAA Journal, 15(1), pp. 24-32, 1977. https://doi.org/10.2514/3.60601

[7] Syngellakis, S., A boundary element approach to buckling of general laminates. WIT Transactions on Modelling and Simulation, 53, pp. 145-155, 2012. https://doi.org/10.2495/be120131

[8] Hwu, C., Green's function for the composite laminates with bending extension coupling. Composite Structures, 63, pp. 283-292, 2004. https://doi.org/10.1016/s0263-8223(03)00175-2

[9] Hwu, C., Boundary integral equations for general laminated plates with coupled stretching-bending deformation. The Royal Society, Proceedings: Mathematical, Physical and Engineering Sciences, 466(2116), pp. 1027-1054, 2010. https://doi.org/10.1098/rspa.2009.0432

[10] Syngellakis, S., Fundamental solutions for the coupled extension-flexure laminate problem. WIT Transactions on Modelling and Simulation, 61, pp. 235-246, 2015. https://doi.org/10.2495/bem380191 
[11] Courant, R. \& Hilbert, D., Methods of Mathematical Physics vol. II: Partial Differential Equations, Interscience Publishers: New York, 1953.

[12] Hildebrand, F.B., Advanced Calculus for Applications, Prentice-Hall, Inc.: Englewood Cliffs, N.J., 1962.

[13] Cheng, Z.-Q. \& Reddy, J.N., Octet formalism for Kirchhoff anisotropic plates. The Royal Society, Proceedings: Mathematical, Physical and Engineering Sciences, 458(2022), pp. 1499-1517, 2002.

https://doi.org/10.1098/rspa.2001.0934 